\title{
The search for Higgs production associated with W, $Z$ with Higgs decaying into WW or tau in CMS
}

\section{Cesare CALABRIA*}

Università degli Studi di Bari “Aldo Moro” and INFN Bari

E-mail: cesare.calabria@cern.ch

\begin{abstract}
At the LHC[1], the Standard Model (SM) Higgs boson is expected to be produced mainly from gluon and vector boson fusion. Higgs production in association with vector bosons $(W H, Z H)$ is $\sim$ one order of magnitude lower. The associated production provides higher background rejection achieved through the additional highly energetic leptons from the $W / Z$ decays. Higgs decays into $\tau$-lepton or $\mathrm{W}$ boson pairs cover the low and intermediate/high Higgs mass range, respectively. For these reasons a search for WH with three-lepton final states is performed based on the data from proton-proton collisions collected with the CMS detector[2] during 2011 and 2012 and corresponding to an integrated luminosity of $4.9 f b^{-1}$ at $\sqrt{s}=7 \mathrm{TeV}$ and $19.5 f b^{-1}$ at $\sqrt{s}=8$ $\mathrm{TeV}$ respectively. A data-driven technique, the fake-rate method, has been used for background estimation. No significant excess of events above the standard model background expectation is observed, so upper limits are set at 95\% CL on the SM Higgs boson production cross-section.
\end{abstract}

The European Physical Society Conference on High Energy Physics

18-24 July, 2013

Stockholm, Sweden

${ }^{*}$ Speaker. 


\section{Final states}

The search for $\mathrm{WH}$ associate production is conducted by using three-lepton final states, depending on the $W$ and $\tau$-lepton decay modes. $W(H \rightarrow \tau \tau)$ channel leads to semi-leptonic $\left(\mu \mu \tau_{\text {had }}\right.$ and $\left.e \mu \tau_{\text {had }}\right)$ or full-hadronic final states $\left(\mu / e \tau_{\text {had }} \tau_{\text {had }}\right)$, where $\tau_{\text {had }}$ indicates a tau hadronic decay. $W(H \rightarrow W W)$ channel leads to $\ell v_{\ell} \ell v_{\ell} \ell v_{\ell}$ final states, where $\ell=e, \mu$.

\section{Physics object selections}

All the three lepton flavors are involved in this study. Muons are reconstructed in the tracker and in the muon chambers. Quality cuts are applied to suppress backgrounds from punch-through and decays in flight. Electrons are reconstructed by combining tracks with ECAL clusters. Charged pions faking electrons and photon conversions are rejected by means of a boosted decision tree multivariate discriminator. Hadronic taus are reconstructed by organizing e.m. objects in "strips" (to take into account photon conversion effects), then the charged hadrons and strips are combined to reconstruct the main hadronic tau decay modes[3, 4]. Muons, electrons and taus are expected to be isolated, for this reason an isolation requirement (properly corrected to take into account pileup effects) is applied. The triplet candidate total charge is required to be \pm 1 . Other topological selections are applied and differ from channel to channel. In the $W(H \rightarrow W W)$ channel the events are categorized in SameSignSameFlavor (SSSF) and OppositeSignSameFlavor (OSSF). Specific missing $E_{T}$ (MET) threshold are applied to suppress $Z \rightarrow \ell \ell+$ jets events. $\Delta R_{\ell^{+} \ell^{-}}$spatial separation in the $\eta-\phi$ plane and cut on $m_{\ell^{+} \ell^{-}}$are also applied. In order to suppress $W Z$ events, the OSSF lepton pairs with mass far from the $Z$ nominal mass are selected[6]. In the $W(H \rightarrow \tau \tau)$ semileptonic final states the softer light lepton is always coupled to the hadronic tau. $Z+j e t s$ and $t \bar{t}$ events are reduced by requiring two same-sign (SS) light leptons. A threshold on the $E_{T}$ scalar sum of the three lepton candidates is applied since the background is expected to have a softer spectrum. In the $W(H \rightarrow \tau \tau)$ full-hadronic final states MET and $M_{T}\left(p_{T}^{\ell}, M E T\right)$ thresholds are applied to reduce $Z \rightarrow \tau \tau$ events and avoid the overlap with $H \rightarrow \tau \tau$ analysis. No opposite-sign (OS) electron pairs or OS $e-\tau_{\text {had }}$ with mass close to the $Z$ nominal mass are allowed. A specific $Z \rightarrow \tau \tau$ veto is also applied[5]. Additional lepton vetoes to further suppress $Z Z, Z+$ jets and a $b$-tagged jet veto to suppress $t \bar{t}$ contributions are common to all the analyses.

\section{Background estimation}

An irreducible contribution to the background comes from multi-boson backgrounds ( $W Z$, $Z Z, Z \gamma, V V V)$. In general their contribution is estimated through the simulation, except for $W Z$ and $Z \gamma$ normalizations in the $W(H \rightarrow W W)$ channel that are data-driven estimated in $W Z$ and $Z+\gamma$ enriched regions respectively. The reducible background contribution is due to "fakes",i.e. background containing at least one quark or gluon jet which is incorrectly identified as an isolated $e, \mu$ or $\tau_{\text {had }}$ and data-driven estimated through the "fake-rate method". First the $j e t \rightarrow \ell$ probability, $f\left(p_{T}, \eta\right)$, is measured with jets in background-enriched regions $(W+j e t s, Z+j e t s, \mathrm{QCD})$ and it is parametrized in terms of jet/tau $p_{T}$ and $\eta$. Then the identification and isolation cuts are inverted on the lepton candidates (one at a time) defining a background enriched region. Each event is 

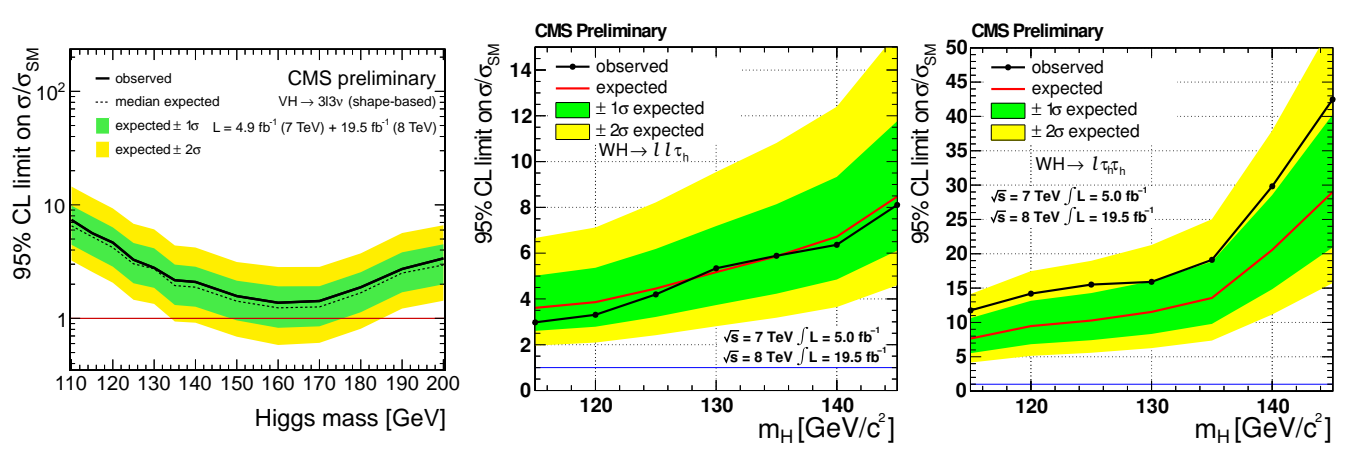

Figure 1: Upper limits at 95\% CL in $24.5 \mathrm{fb}^{-1}$ in the SM Higgs scenario for the $W(H \rightarrow W W)$ shapebased analysis (left)[6] and the $W(H \rightarrow \tau \tau)$ semi-leptonic (center) and full-hadronic (right) analyses[5]. The expected limits in the presence of a SM Higgs with $\mathrm{m}_{\mathrm{H}}=125 \mathrm{GeV} / \mathrm{c}^{2}$ are also shown.

then weighted by $w_{i}\left(p_{T, i}, \eta_{i}\right)=f\left(p_{T, i}, \eta_{i}\right) /\left(1-f\left(p_{T, i}, \eta_{i}\right)\right)$, i.e. the probability for a jet to pass the inverted cuts and so its contribution to the signal region[5, 6]. The main systematic uncertainty for all the analyses comes from the fake-rate technique. In the $W(H \rightarrow \tau \tau)$ channel the sources of systematics are the fake-rate probability dependency on the specific process and the fake-rate function fit uncertainty. A total systematic uncertainty of $15-30 \%$ is assigned to the semi-leptonic final states and $20 \%$ to the full-hadronic final states[5]. In the $W(H \rightarrow W W)$ channel the fake-rate shows a dependency on the jet $p_{T}$ threshold that leads to a $\sim 30 \%$ uncertainty. A further $\sim 20 \%$ is added due to the closure test on a QCD MC sample. An overall scale factor from a top-enriched region is used for calibrating the background prediction[6].

\section{Final results and exclusion limits}

No significant excess of events above the SM background expectation is observed in the physics observable distributions used in each analyses. The observation is consistent both with the presence and the absence of a SM Higgs boson. Upper limits at 95\% C.L. are established in all the three channels (figure 1) on the product of Higgs boson cross-section and the specific decay branching fraction for Higgs masses between 110 and $200 \mathrm{GeV} / \mathrm{c}^{2}$. At $125 \mathrm{GeV} / \mathrm{c}^{2}$ observed limits are between $\sim 3$ and $\sim 15$ times the Standard Model prediction[5, 6].

\section{References}

[1] Lyndon Evans and Philip Bryant LHC Machine J. Instrum. 3 (2008)

[2] CMS Collaboration. The CMS experiment at the CERN LHC J. Instrum. 3 (2008).

[3] CMS Collaboration. Particle-Flow Event Reconstruction in CMS and Performance for Jets, Taus, and MET. CMS-PAS-PFT-09-001

[4] CMS Collaboration. Performance of $\tau$-lepton reconstruction and identification in CMS. J. Instrum. 7 (2012) P01001

[5] CMS Collaboration. Search for the standard model Higgs boson decaying to tau pairs produced in association with a $W$ or $Z$ boson $C M S-P A S-H I G-12-053$

[6] CMS Collaboration. Search for SM Higgs in WH to WWW to 313nu CMS-PAS-HIG-13-009 